\title{
Influence of geometrical imperfection on critical temperature evaluation for steel corrugated arch sheets exposed to fire
}

\author{
Mariusz Maślak ${ }^{1, *}$, Michał Pazdanowski ${ }^{1}$, and Maciej Suchodoła ${ }^{1}$ \\ ${ }^{1}$ Cracow University of Technology, Warszawska 24, 31-155 Cracow, Poland
}

\begin{abstract}
The critical temperature for an arched corrugated steel sheet exposed to fully developed fire is determined in this paper. The analysis is performed on 3D numerical model developed within the ANSYS computational environment with parameters calibrated experimentally on an experiment performed for persistent design scenario. During the simulations performed the self-supporting steel sheet with an assumed initial shape imperfection is at first loaded with static load, up to a predetermined level of material effort, and subsequently evenly heated until the capability to safely resist the applied load is completely exhausted. Because of the nonlinearity of the analysis having several sources, the location of the limit point on the equilibrium path determined during calculations represents the temperature sought. Three imperfection patterns, denoted as A, B and C are considered. In the case A the imperfection has the shape and magnitude measured in situ, selected as representative for a set of 10 steel sheets of the same type. In the case B the imperfection is a substitute one with size analogous to that assumed in the case A, but with a different shape, corresponding to the first antisymmetric buckling mode identified in conventional Local Buckling Analysis (LBA). In the case C the shape of imperfection B has been kept, but the positive and negative deviation from the perfect circular shape have been increased twofold.
\end{abstract}

\section{Introduction}

Self-supporting arched corrugated sheet metal panels made of steel constitute the subject of our analysis. Behaviour of such components when subjected to load is highly nonlinear, wherein the persistent design scenario the nonlinearity is mainly of geometrical nature taking into account the imperfect geometry of the considered panel. The bearing capacity of the panel is, in such case, determined by the location of the limit point on the nonlinear static equilibrium path specified in the load displacement coordinate system [1]. If the symmetric load is applied to the ,perfect” panel, with perfect geometry, then after application of limit load the bifurcation of possible equilibrium configurations occurs [2]. However, in practical applications the considered sheet metal panels exhibit usually large and nonsymmetric geometrical imperfections. The shape and size of those imperfections determine the behaviour of considered structural elements when subjected to load [3]. The experiments performed on arched and initially deformed corrugated steel panels under the assumption of persistent design situation confirmed their exceptional sensitivity to various forms of stability loss - not only the global stability loss related to the whole panel, but also the local one with limited reach [4]. In the paper [5] it was shown, that a global loss of stability in this kind of profile is related to the formation of local instabilities in the early stages of local plastic mechanisms development. Instability of this type in many cases implies the destruction of the whole bearing structure made of such panels - as was thoroughly investigated during the analysis of failed steel halls erected using ABM system [6].

The computer simulations of corrugated sheet metal panel behaviour when subjected to load have been performed so far only for the typical scenario of the persistent design situation and thus did not take into account the potential additional action of fire temperature [7]. In this paper we would like to verify the influence of size and shape of the considered imperfections on the critical temperature specified for arched corrugated sheet metal panels made of steel, and supporting uniformly distributed gravitational loads, while subjected to fire exposure. The scenario in which the panel was at first statically loaded up to a certain level of material effort, and subsequently under constant static load uniformly heated with increasing intensity is considered here. The panel was heated until its capability to safely resist the loads applied to it was completely exhausted. The response of the considered sheet metal panel to the load application sequence described above was simulated numerically within the ANSYS computational environment [8], while the parameters of the computational model had been previously calibrated based on the independently performed experiment. The pattern of imperfections assumed for calibration, with respect to both assumed imperfect panel shape, as well as the representative deviations from the perfect geometry correspond to real imperfections measured on

* Corresponding author: mmaslak@pk.edu.pl 
a set of 10 panels of this type available for detailed inspection.

\section{Description of experiment}

\subsection{Characteristics of analysed panels}

The experimental results obtained independently for analogous panels loaded only in a static mode, according to the rules of persistent design scenario have been used to verify the bearing capacity of considered panels when subjected to the fully developed fire conditions. In those experiments ten arched sheet metal panels having the perfect reference geometry of circular arch, dimensions juxtaposed in Table 1 and the cross section depicted in detail in Fig. 1 have been used. The interpretation of denotations used in Table 1 is shown in Fig. 2. The table contains as well the values of geometrical imperfections, in each case corresponding to the vertical distances between the actual panel axis and the ,perfect” panel axis measured at points $\mathrm{A}, \mathrm{B}$ and $\mathrm{C}$ located at $1 / 4,3 / 4$ and $1 / 2$ of arch span, respectively.

Table 1. Dimensions of sheet metal panels used in the experiment and vertical deviations of the real geometry from the perfect circular shape measured at points A, B and C, modeling the geometrical imperfections.

\begin{tabular}{|c|c|c|c|c|c|}
\hline \multirow{2}{*}{ Panel No. } & \multicolumn{2}{|c|}{ Perfect arch geometry } & \multicolumn{3}{|c|}{ Measured imperfections } \\
\cline { 2 - 6 } & $\mathbf{L}[\mathbf{m m}]$ & $\mathbf{R}[\mathbf{m m}]$ & $\boldsymbol{\Delta}_{\mathbf{A}}[\mathbf{m m}]$ & $\boldsymbol{\Delta}_{\mathbf{B}}[\mathbf{m m}]$ & $\boldsymbol{\Delta}_{\mathbf{C}}[\mathbf{m m}]$ \\
\hline 1 & 8734 & 8390 & 15,8 & $-1,0$ & 2,9 \\
\hline 2 & 8278 & 8000 & 23,4 & 2,2 & 7,1 \\
\hline 3 & 10580 & 11400 & $-1,4$ & $-12,9$ & $-2,7$ \\
\hline 4 & 7840 & 11518 & 0,8 & $-8,3$ & $-2,6$ \\
\hline 5 & 7420 & 15175 & 0,9 & $-7,6$ & $-3,5$ \\
\hline 6 & 8368 & 8000 & $-7,6$ & $-7,8$ & $-3,5$ \\
\hline 7 & 6200 & 8000 & 7,5 & $-1,2$ & 6,8 \\
\hline 8 & 6200 & 8000 & $-5,3$ & $-17,9$ & $-14,9$ \\
\hline 9 & 6200 & 8000 & 3,0 & $-13,0$ & $-5,2$ \\
\hline 10 & 6200 & 8000 & 10,8 & 9,3 & 0,3 \\
\hline
\end{tabular}

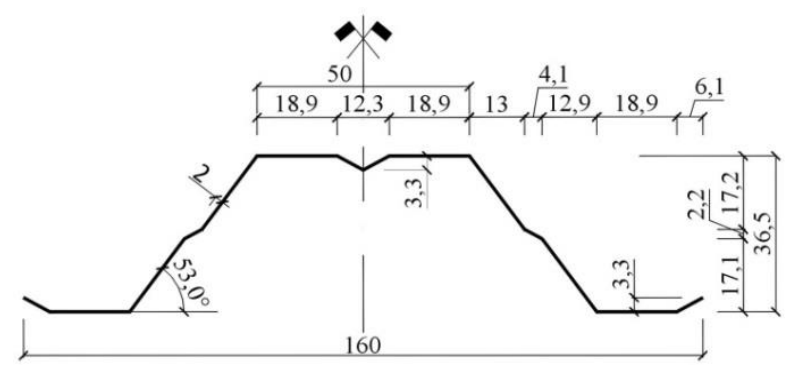

Fig. 1. Repetitive part of the corrugated sheet metal panel cross section as considered in the paper.

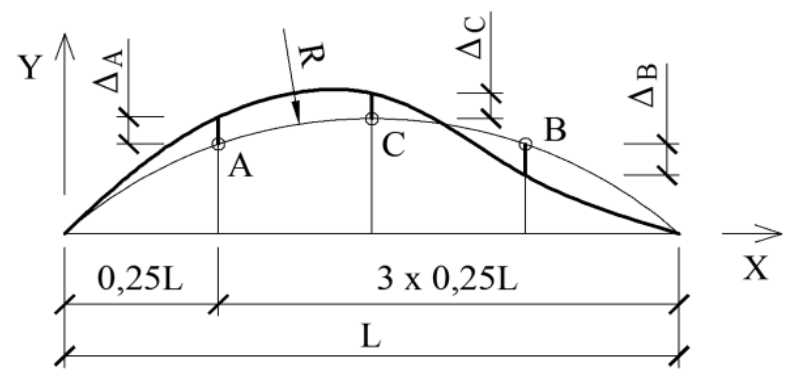

Fig. 2. Panel dimensions for analyzed panels listed in the Table 1 and location of the measurement points determining the shape of the assumed geometrical imperfection.

Results of the measurements listed in the Table 1 allowed for assignment of a specific imperfection pattern to each of the considered sheet metal panels. The shapes of these imperfect panels are depicted in Fig. 3. The shape of panel No. 10 (see Table 1) has been selected for further analysis as the representative for the whole set. This pattern will be in all following considerations denoted as geometrical imperfection of the type A.
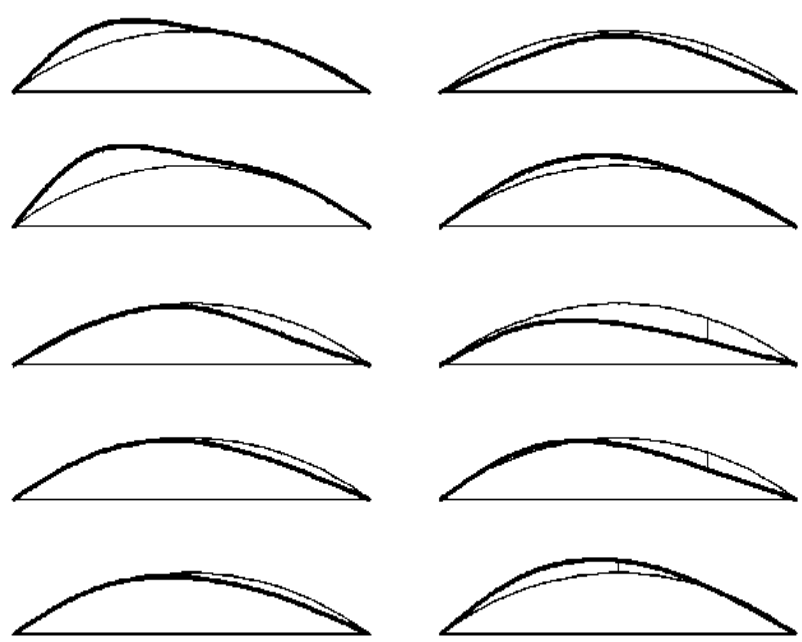

Fig. 3. The panel imperfection patterns identified based on the measurements listed in Table 1. Panels 1 to 5 are depicted at left from the top, while panels 6 to 10 are depicted at right from the top. 


\subsection{Description of the experimental setup}

The experiments have been performed on single or double panels (Table 1), having the width of $960 \mathrm{~mm}$ in each panel. In order to model the flexibility of the supports with respect to horizontal displacements, a tie connecting supports at both has been applied. This tie was made of two steel cables, located in parallel to each other and hooked to rigid beams mounted at both ends of sheet metal panels.

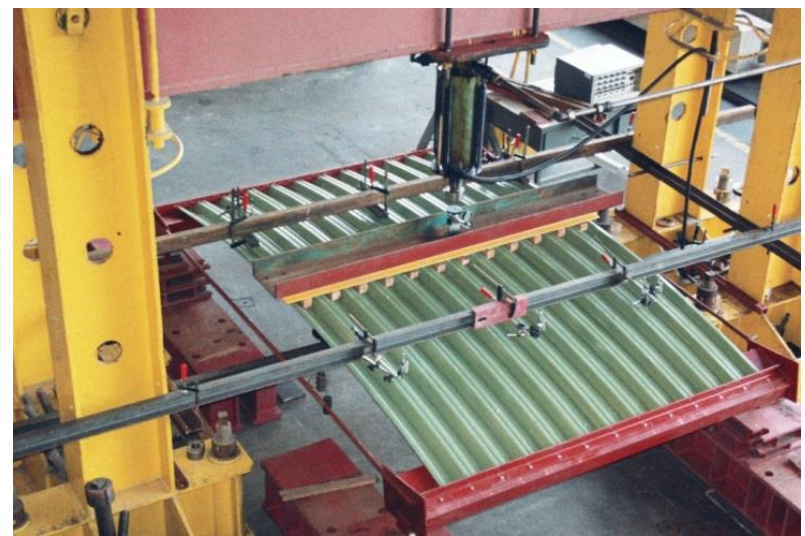

Fig. 4. Experimental setup with double arched sheet metal panel. The tie and deformation measurement method are visible. The load is applied at the arch crown (point $\mathrm{C}$ in Fig. 5a).

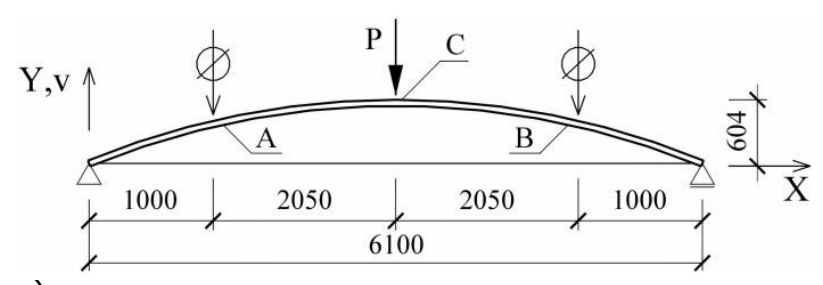

a)

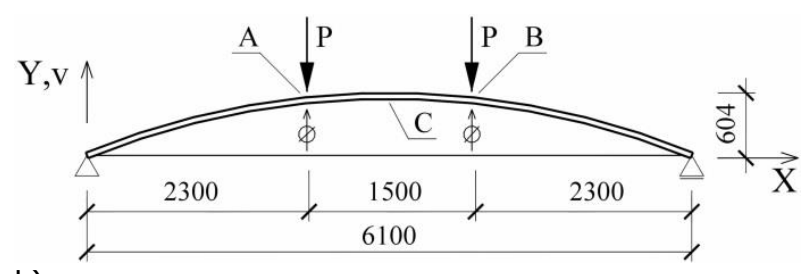

b)

Fig. 5. Load application methods implemented in series of measurements and location of points in which the deformations of sheet metal panels have been measured: a) load applied only in the arch crown (point C) - first set of measurements, b) load applied in the points A and B located symmetrically with respect to the arch crown - second set of measurements.

Because of practical considerations the static load has been applied only via sufficiently rigid transversal beams - this resulted in loads distributed evenly only along the sheet metal panel width (Fig. 4). The static load had been gradually increased in each case until the limit point on the equilibrium path has been reached. Two series of experiments have been conducted. During the first series the load has been applied only in the crown of the arch (Fig. 5a), while during the second series the load has been applied in the points $\mathrm{A}$ and $\mathrm{B}$ distributed symmetrically with respect to the arch crown, as shown in Fig. 5b. It has to be noted, however, that the location of points $\mathrm{A}$ and $\mathrm{B}$ during the experiment and subsequent numerical simulations did not coincide (Fig. 2). In addition, in order to properly shape the supports, the actual arch span during the experiment was somewhat lower than the span of the unrestrained sheet metal panels measured on the ground and given in the Table 1 (for instance in the case of panels no. 7 through 10 instead of the initial $6200 \mathrm{~mm}$ only $6100 \mathrm{~mm}$ has been obtained, as shown in Fig. 5a and 5b.

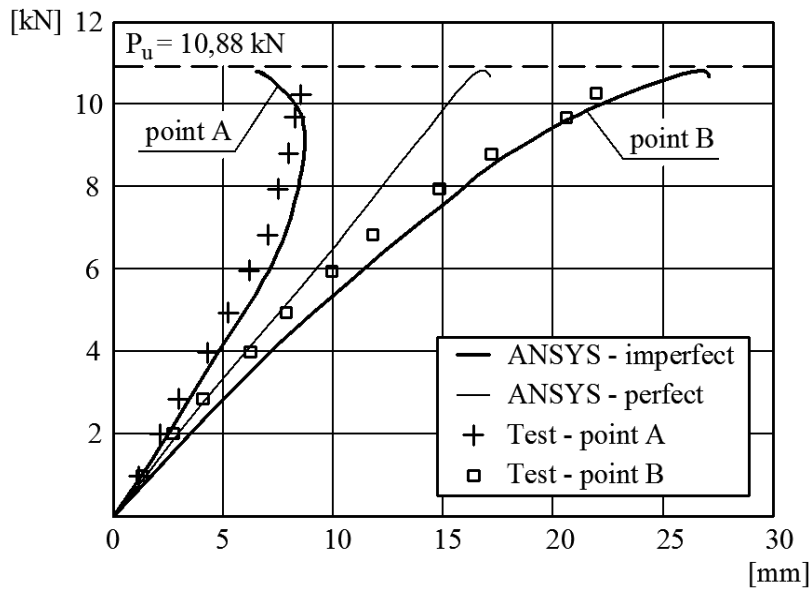

a)

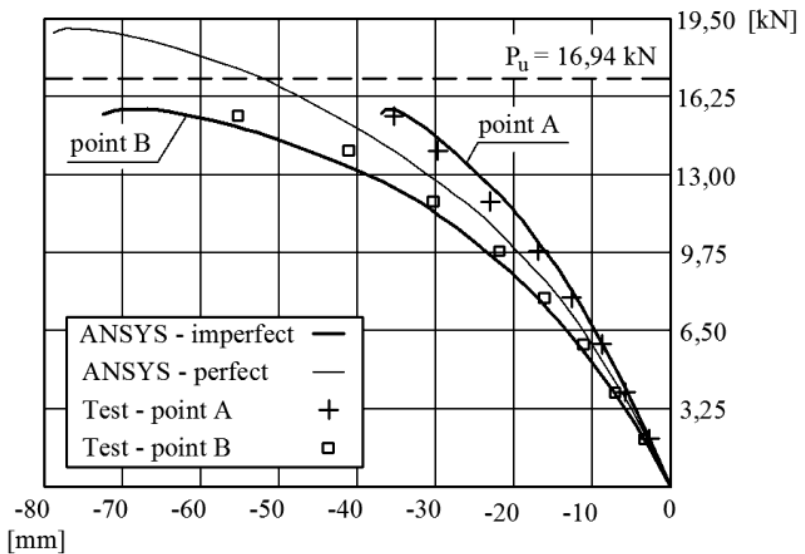

b)

Fig. 6. Experimental and numerically simulated static equilibrium paths for sheet metal panel no. 10 of Table 1: a) obtained at the load depicted in Fig. 5a, b) obtained at the load depicted in Fig. 5b.

\subsection{Static equilibrium paths obtained experimentally for the sheet metal panel no. 10}

Detailed presentation of results obtained experimentally is limited here only to those specific to the sheet metal panel no. 10 (see Table 1). In authors' opinion these results may be considered representative for the whole analysed population of panels. Fig. 6a depicts the points marking out the static equilibrium paths obtained for the load distribution depicted in Fig. 5a, while Fig. 6b depicts the points marking out the static equilibrium paths obtained for the load distribution depicted in Fig. 5 b. The crosses pertain to the force - displacement 
relationship identified at point $A$, located to the left of crown, while the circles pertain to the analogous relationships at point $\mathrm{B}$, located to the right of crown. One may easily observe, that when the increasing load is applied at the crown only (Fig. 6a) and directed vertically downwards, the points $\mathrm{A}$ and $\mathrm{B}$ are subjected to progressive uplift. Thus both equilibrium paths are slanted rightwards, but the speed of the uplift at point B steadily increases, while at the point A it gradually slows down. However, should one consider the situation depicted in Fig. 6b, when downward oriented forces are applied at points $\mathrm{A}$ and $\mathrm{B}$ at the same time, it would be obvious, that in such case these points would move downwards with increasing intensity. Thus both equilibrium paths in this case are slanted leftwards, and the speed of the downward movement during the whole experiment gradually increases.

\section{Numerical simulation of the sheet metal panel behaviour in case of fire}

\subsection{Verification of applicability of the numerical model}

Numerical simulation of the behaviour of considered sheet metal panels when subjected to the fully developed fire has been performed within the ANSYS computational environment [4], on a 3D model made of shell elements of the type shell181 and depicted in Fig. 7. An elastic - plastic material model has been assumed for the structural steel of which the analysed panels have been made, in agreement with the experimentally determined stress - strain relationship depicted in Fig. 8. Based on this figure the authoritative yield limit for the modelled steel has been assumed at $f_{y}=413 M P a$, while the kinematic hardening parameter has been set at $E_{t} / E=1 / 10000$.

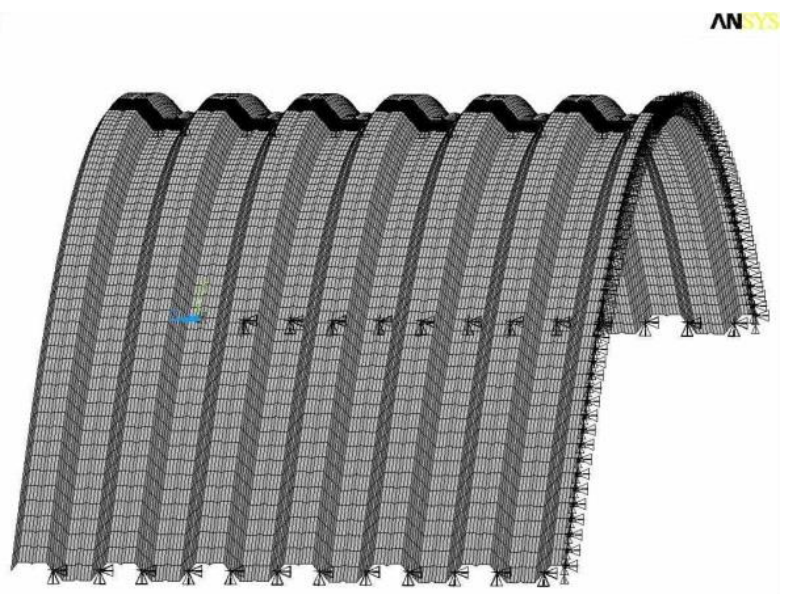

Fig. 7. Geometry, meshing and support conditions applied in the numerical model used by the authors to simulate the behaviour of considered panels when subjected to fully developed fire conditions.

Before beginning the simulation of the considered panels behaviour when subjected to the fully developed fire conditions, it had to be verified whether the applied numerical model was sufficiently well calibrated. In order to do that, verification simulations have been performed to forecast the deformation of the sheet metal panel no. 10 with precisely replicated shape imperfection measured on the actual panel before the experiment, and registered after application of the monotonically increasing static load corresponding to the schemes depicted in Fig. 5a and 5b.

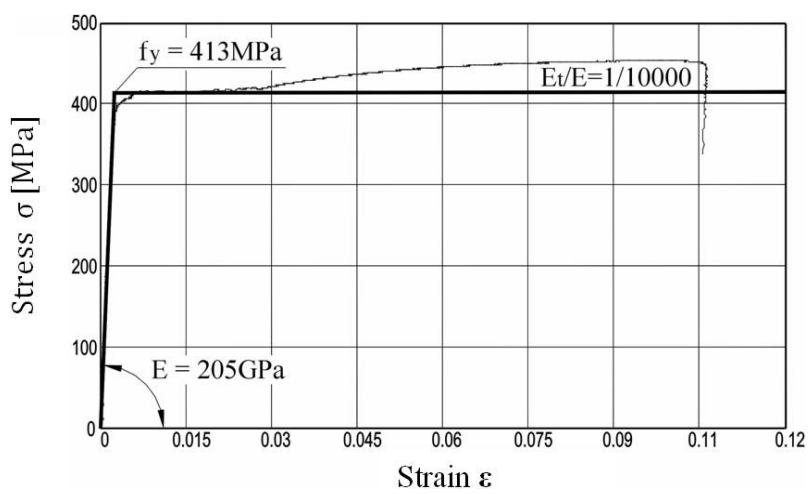

Fig. 8. Experimentally determined characteristics of the structural steel, of which the sheet metal panels have been made adapted to numerical model.

Static equilibrium paths obtained during these simulations have been overlaid in Fig. $6 a$ and $6 \mathrm{~b}$ with points obtained during previously conducted experiments. Both graphs at the same time have been enriched with presentation of equilibrium paths determined for analogous but ,perfect” panels, having perfect geometry. In authors' opinion the comparisons quoted above indicate good convergence of the results obtained numerically with those obtained during the experiment. Based on that we dare to say, that the prepared numerical model is fully applicable to the case of search for equilibrium paths typical for the fully developed fire scenario.

\subsection{Changes in the size and shape of geometrical imperfection measured on panel no 10 subjected to the simulated fully developed fire}

Fully developed fire denotes action of a uniform temperature field of increasing intensity on the statically loaded sheet metal panel. This is equivalent to the assumption, that in the direct vicinity of said panel a plume of hot exhaust gases of even, but increasing in time, temperature is present. This in turn translates into the uniform and increasing in time steel temperature at each and every point of the panel. The increase in panel temperature results in the thermal elongation of the panel, and this in turn with restrained capability for horizontal displacements at the supports, results in additional buckling of the arch. With perfect geometry (panel of the ,perfect” type), this buckling would remain symmetric. However, the presence of initial imperfection (panel of the ,imperfect" type) results in uneven buckling, depending on the imperfections measured earlier in the room (reference) temperature. In the case of the sheet metal panel no. 10, analysed here in detail, the 
values of vertical coordinates describing the location of the deformed panel axis at points $\mathrm{A}, \mathrm{B}$ and $\mathrm{C}$, as shown in Fig. 2, are listed in the Table 2 for various temperature values $\theta\left[{ }^{\circ} \mathrm{C}\right]$. The differences between those coordinates, specified for the fire conditions and "perfect" as well as "imperfect" panels, are interpreted by the authors as generalized arch shape imperfection measure for the considered sheet metal panel. Let us note, that during the simulated fire not only the distance values for the previously identified imperfection pattern change with increasing temperature at the considered points, but also the shape of the pattern itself undergoes transformation, and this in turn affects the forecast deformation of the heated panel thus affecting its effective bearing capacity. Detailed data of this type, obtained after analysis of the panel no. 10 are juxtaposed in the Table 3.

Table 2. Numerically simulated for sheet metal panel no.10 location of the deformed panel axis in the case of simulated fire conditions - panel devoid of imperfections (,,perfect” type) and corresponding imperfect panel (,imperfect” type).

\begin{tabular}{|c|c|c|c|c|c|c|}
\hline$\theta\left[{ }^{\circ} \mathrm{C}\right]$ & $v_{A}^{\text {perf }}[\mathrm{mm}]$ & $v_{B}^{\text {perf }}[\mathrm{mm}]$ & $v_{C}^{\text {perf }}[\mathrm{mm}]$ & $v_{A}^{\text {imp }}[\mathrm{mm}]$ & $v_{B}^{\text {imp }}[\mathrm{mm}]$ & $v_{C}^{\text {imp }}[\mathrm{mm}]$ \\
\hline 200 & 2,169 & 2,169 & 3,019 & 2,132 & 2,144 & 3,004 \\
\hline 400 & 4,452 & 4,452 & 6,331 & 4,430 & 4,453 & 6,246 \\
\hline 600 & 7,031 & 7,031 & 9,823 & 6,874 & 6,910 & 9,702 \\
\hline
\end{tabular}

Table 3. Imperfections of arch shape for panel no. 10, simulated at points A, B and C under assumption of fire exposure.

\begin{tabular}{|c|c|c|c|c|}
\hline$\theta\left[{ }^{\circ} \mathrm{C}\right]$ & $\Delta_{A}^{\theta}[\mathrm{mm}]$ & $\Delta_{B}^{\theta}[\mathrm{mm}]$ & $\Delta_{C}^{\theta}[\mathrm{mm}]$ & $\Delta_{B}^{\theta}-\Delta_{A}^{\theta}[\mathrm{mm}]$ \\
\hline 20 & 0,033 & 1,076 & 0,935 & 1,043 \\
\hline 200 & $-0,004$ & 1,051 & 0,920 & 1,055 \\
\hline 400 & 0,011 & 1,077 & 0,850 & 1,067 \\
\hline 600 & $-0,124$ & 0,955 & 0,814 & 1,079 \\
\hline
\end{tabular}

As can be observed in this case, with increasing panel temperature, besides obvious thermally induced buckling an additional change in the arch shape has been observed. Point B for the panel with imperfections is always located markedly above the corresponding location of the same point at the same temperature but for the panel devoid of imperfections. Similar situation occurs at point $\mathrm{C}$, but in this case the difference in location is not so pronounced. However, the same conclusion may not be drawn in the case of point A. Here interesting fluctuations of the location are observed. In each of the considered cases these differences are relatively small and seem to be negligible from the point of view of practical safety evaluation in fire conditions.

\subsection{Static equilibrium paths simulated for the panel no. 10 heated in fire}

The detailed equilibrium paths for sheet metal panel no. 10 have been numerically simulated for fire conditions under the assumption that the panel geometry is imperfect and prior to the fire exposure the panel had been loaded up to a predetermined level by a downwardly oriented vertical load of the gravitational type, evenly distributed over the whole panel area. Thus the static load had been applied in a manner different than the manner used during the experiments described above. Taking into account the thermal load of increasing intensity applied after the static load allowed for the determination of the critical temperature for the considered panel. This temperature is correlated with the panel losing the capability to safely resist the loads applied to it. The static equilibrium paths obtained for the fire scenario are presented in the coordinate system of sheet metal panel temperature - vertical displacement at the selected point on the surface. Equilibrium paths of this type obtained at points $\mathrm{A}$ and $\mathrm{B}$ of the considered sheet metal panel, under the assumption that the panel is statically loaded up to $60 \%$ of the limit load before initiation of fire exposure are depicted in Fig. 9.

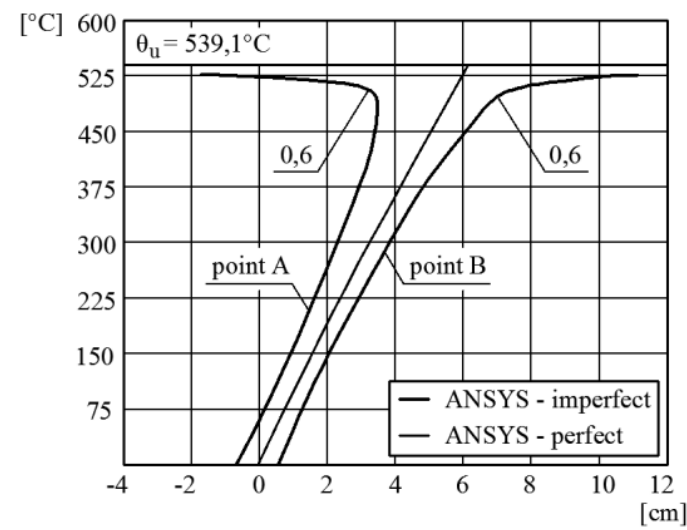

Fig. 9. Static equilibrium paths obtained in computer simulation for sheet metal panel no. 10 with perfect and imperfect geometry, exposed to fire conditions, under the assumption that the static load prior to fire was limited to $60 \%$ of limit load.

The analysis of a panel with perfect geometry yields a quasi-linear equilibrium path ending in bifurcation of equilibrium state. When a shape imperfection existing prior to fire initiation is taken into account the equilibrium paths determined at points $\mathrm{A}$ and $\mathrm{B}$ of the considered panel become divergent. Those paths are now 
clearly nonlinear. Let us note, however, that in the presented example the bearing capacity of the considered sheet metal panel, as determined by the location of limit point on the appropriate equilibrium path, and estimated at $\theta_{u}=539,1^{\circ} \mathrm{C}$, does not substantially disagree with an analogous value obtained after the analysis of a panel devoid of any imperfections.

The dependence of the static equilibrium paths at points A and B of sheet metal panel no. 10 with assumed imperfections on the level of material effort prior to fire initiation is depicted in Fig. 10. A quite obvious conclusion follows, that the higher the material effort prior to fire initiation, the lower the final critical temperature $\theta_{u}$. This is undoubtedly a consequence of decreasing reserve bearing capacity, which may be used during fire. However, such result does not mean that the bearing capacity of the sheet metal panel is reduced in fire conditions, as during the specification of such bearing capacity the influences of both types of action static and thermal - have to be added.

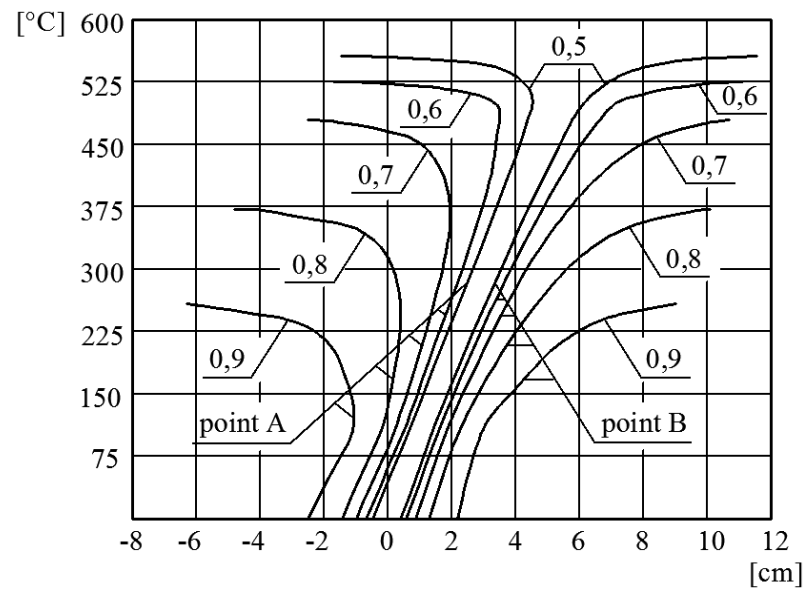

Fig. 10. Dependence of the static equilibrium paths determined at points A and B of sheet metal panel no. 10 with assumed shape imperfection on the level of material effort prior to initiation of fire action.

\section{Fire bearing capacity of panel no. 10 with substitute antisymmetric shape imperfection}

\subsection{Imperfection of value identical with the one measured during experiment}

In this computational approach the real imperfection has been replaced by the substitute imperfection exhibiting the same magnitude of deviation from the perfect shape, but a different shape, conforming to the first antisymmetric buckling mode determined during conventional Linear Buckling Analysis. In the following analyses this imperfection would be denoted as the B type imperfection. Thus at point $\mathrm{A}$ of the considered panel the deviation is of negative sign, while at point B it is of positive sign, as determined with respect to the axis of a perfect panel. According to the authors' opinion this shape of imperfection seems to most adversely affect the bearing capacity of a sheet metal panel in fire, and thus accounts for the influences of all potentially possible geometrical imperfections, not only those depicted above in Fig. 3

The equilibrium paths analogous to those depicted in Fig. 10, but obtained during numerical simulations performed for sheet metal panel no. 10 with assumed substitute shape imperfection of a magnitude and shape described above are juxtaposed in Fig. 11.

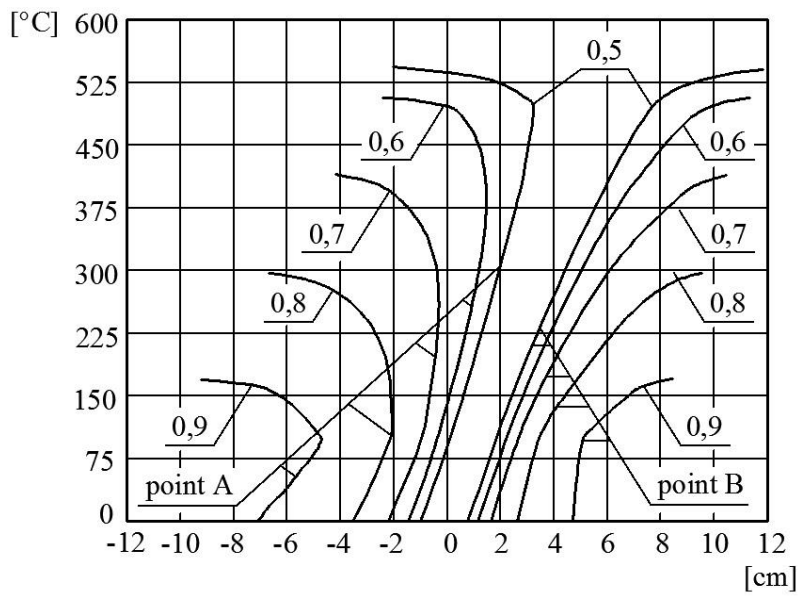

Fig. 11. Static equilibrium paths obtained at points $A$ and $B$ of sheet metal panel no. 10 with assumed shape imperfection, analogous to those depicted in Fig. 10, but determined for the substitute antisymmetric shape imperfection exhibiting the positive and negative deviation from perfect shape corresponding to the deviation measured in experiment.

A simple quantitative comparison of the corresponding limit points correlated with static equilibrium paths specified at the same level of material effort attained before fire initiation leads to the conclusion, that simple change of the imperfection shape, not accompanied by changes in the magnitude of deviations from perfect panel shape results in very substantial reduction of the critical temperature $\theta_{u}$ estimated during numerical simulation. The appropriate juxtaposition is listed in the Table 4 of this paper. Quantitative comparison is especially authorized here, as in Fig. 10 as well as in Fig. 11 the limit load level $q_{\text {imp }, u}$ has been determined in the same manner, i.e. under the assumption that the geometrical imperfection of the type $\mathrm{A}$ is modeled.

\subsection{Antisymmetric substitute imperfection with deviation doubled with respect to the experiment}

For comparative purposes the numerical simulations have been performed for sheet metal panel no. 10 with substitute antisymmetric shape imperfection of magnitude doubled with respect to the one analyzed in detail in the chapter 4.1 of this paper. In the following considerations this imperfection would be denoted as the $\mathrm{C}$ type. The authors intended to check how the critical temperature of a sheet metal panel having an imperfect geometry and subjected to the same sequence of at first static and subsequently thermal load would be affected by the magnitude of the imperfection determined at the 
same points of the considered panel. It has to be noted here, that this imperfection pattern, very dangerous from the point of view of global safety analysis resulted in the material effort grossly exceeding the bearing capacity of the considered structure. The static load determined at the level of $90 \%$ of limit load for an imperfect panel exhibiting the A type imperfection proved to be to high to be safely supported by the panel exhibiting the substitute $\mathrm{C}$ type imperfection even at the room temperature. Thus this level of material effort is not represented in the juxtaposition of static equilibrium paths obtained for the so defined substitute imperfection (of $\mathrm{C}$ type), depicted in detail in Fig. 12.

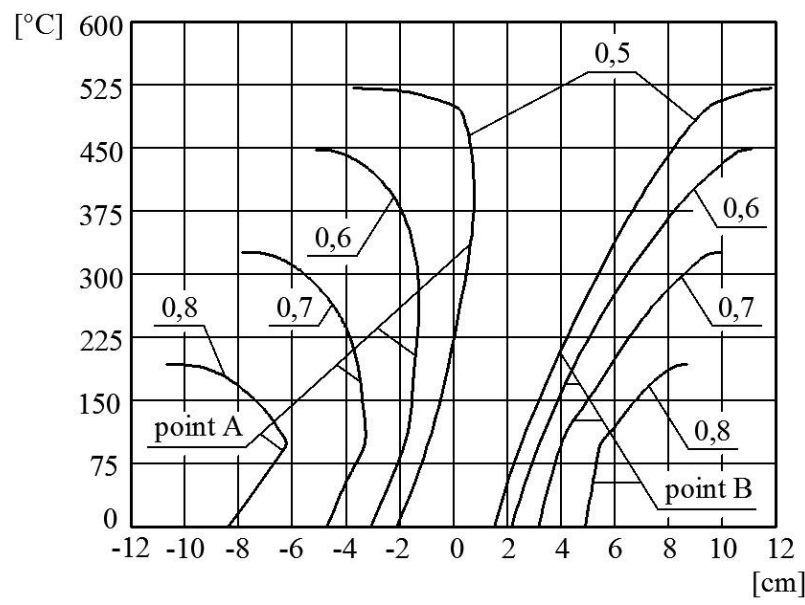

Fig. 12. Static equilibrium paths obtained at points A and B of sheet metal panel no. 10 with assumed shape imperfection, analogous to those depicted in Fig. 10 and 11, but determined for the substitute antisymmetric shape imperfection exhibiting the positive and negative deviation from perfect shape corresponding to the doubled deviation measured in experiment.

Analysis of equilibrium paths depicted in Fig. 12 and comparison with the analogous paths depicted in Fig. 10 and 11 allows for conclusion, that further substantial reduction in the estimated critical temperature of considered sheet metal panel is observed. The detailed juxtaposition of the critical temperature related to the assumed imperfections of the $\mathrm{A}, \mathrm{B}$ and $\mathrm{C}$ type is given in the Table 4. Let us note as well, that the ,spread" of an equilibrium paths set specified for points $\mathrm{A}$ and $\mathrm{B}$ of the same sheet metal panel exhibiting imperfect geometry, and depicted in sequence in Fig. 10, 11 and 12 obviously grows with increasing influence exerted by the modeled imperfection.

\section{Concluding remarks}

According to the authors' opinion the static equilibrium paths determined for thin-walled corrugated sheet metal panels made of steel, exhibiting imperfections and exposed to fire conditions constitute an efficient computational tool which may be used to reliably determine the critical temperature of such panels. The critical temperature is correlated here with capability to safely resist the external loads applied to those panels. The paths of this type are specified in the coordinate system of sheet metal panel temperature - vertical displacement of a selected point on the panel surface. Thus the critical temperature is found as the maximum panel temperature, which may be associated with the determined static equilibrium path. Obviously, the shape of the equilibrium path depends on the location of the point for which it is determined, but the asymptote of this path does not change with changing location of the point, and thus this asymptote does determine the limit value of the sought temperature. The content of the Table 4 indicates, that the geometrical imperfection of the panel arch substantially affects the authoritative value of the critical temperature. This influence grows with decreasing reserve bearing capacity of the considered panel determined before initiation of the fire exposure. The comparisons performed indicate as well the existence of an unequivocal dependence between the critical temperature determined for the considered sheet metal panel and substitute geometrical imperfection pattern assumed for this panel. This dependence holds for both the shape and magnitude of the imperfection. In general one may state that the more adversely the imperfection is modeled the more conservative the final estimate of the critical temperature is. An appropriate juxtaposition of the critical panel temperature values determined for the same sheet metal panel no. 10 having imperfect geometry, with substitute shape imperfections of the type A, B and C, respectively, is shown in the Table 4. However, one should keep in mind that there exists only an indirect coupling between the critical temperature specified for selected sheet metal panel in a manner described in this paper, and the bearing capacity of the same panel reduced due to the fire conditions. The second of these two values represents the measure of the resistance the considered panel may present to the combined actions of static and thermal loads, while the first one may be estimated only as high as the reserve panel bearing capacity, remaining after application of the static load.

Table 4. Influence of the material effort level in sheet metal panel no. 10 identified before the initiation of fire exposure and assumed substitute geometrical imperfection (A, B or C) on the final estimate of the critical temperature.

\begin{tabular}{|c|c|c|c|c|}
\hline$q / q_{i m p, u}$ & $\begin{array}{l}\theta_{c r}^{\text {perf }} \\
{\left[{ }^{\circ} \mathrm{C}\right]}\end{array}$ & $\begin{array}{l}\theta_{c r}^{\text {impA }} \\
{\left[{ }^{\circ} \mathrm{C}\right]}\end{array}$ & $\begin{array}{l}\theta_{c r}^{\text {impB }} \\
{\left[{ }^{\circ} \mathrm{C}\right]}\end{array}$ & $\begin{array}{l}\theta_{c r}^{\text {impC }} \\
{\left[{ }^{\circ} \mathrm{C}\right]}\end{array}$ \\
\hline 0,5 & 568,2 & 557,5 & 543,0 & 521,6 \\
\hline 0,6 & 539,1 & 525,8 & 508,0 & 448,2 \\
\hline 0,7 & 509,2 & 479,6 & 416,0 & 326,4 \\
\hline 0,8 & 430,2 & 372,5 & 299,2 & 192,9 \\
\hline 0,9 & 326,3 & 258,6 & 171,5 & - \\
\hline
\end{tabular}

\section{References}

1. A. Piekarczuk, K. Malowany, P. Więch, M. Kujawińska, P. Sulik, Stability and bearing capacity of arch-shaped corrugated shell elements. 
Experimental and numerical study, Bulletin of the Polish Academy of Sciences. Technical Sciences, 63, 1, 113-123 (2015)

2. P. Casariego, M. Casafont, J. Muñoz, A. Floreta, M. Ferrer, F. Marimon, Failure mechanisms of curved trapezoidal steel sheeting, Proceedings of the 6th European Conference on Steel and Composite Structures "EUROSTEEL 2011", Budapest, August 31 - September 2, 2011, A, 63-68 (2011)

3. M. Gwóźdź, M. Suchodoła M., Load-bearing capacity of corrugated arch sheets with imperfections, Archives of Civil Engineering, 54, 1, 147-166 (2008)

4. R. Cybulski, R. Walentyński, M. Cybulska, Local buckling of cold-formed elements used in arched building with geometrical imperfections, Journal of Constructional Steel Research, 96, 1-13 (2014)

5. A. Piekarczuk, P. Więch, K. Malowany, Numerical investigation into plastic hinge formation in arched corrugated thin-walled profiles, Thin-Walled Structures, 119, 13-21 (2017)

6. A. Biegus, A. Kowal, Collapse of halls made from cold-formed steel sheets, Engineering Failure Analysis, 31, 189-194 (2013)

7. A. Piekarczuk, K. Malowany, Comparative analysis of numerical models of arch-shaped steel sheet sections, Archives of Civil and Mechanical Engineering, 16, 645-658 (2016)

8. P. Kohnke (Ed.), ANSYS ${ }^{\circledR}$ : Theory reference. Release 5.6. Canonsburg, PA, USA, November 1999. 